\title{
Intracameral gnathostomiasis: a cause of anterior uveitis and secondary glaucoma
}

\author{
SOMCHAI KITTIPONGHANSA,' ARPORN PRABRIPUTALOONG,' \\ SUCHART PARIYANONDA,$^{2}$ AND ROBERT RITCH ${ }^{3}$
}

From the Departments of 'Ophthalmology and '2 Parasitology, Faculty of Medicine, Srinagarind Hospital, Khon Kaen University, Khon Kaen, Thailand, and the ${ }^{3}$ Department of Ophthalmology, the New York Eye and Ear Infirmary, New York, USA

SUMMARY Recognition of parasitic diseases by Western physicians is becoming increasingly important because of increased international travel and the recent influx of refugees from South east Asia. We describe two patients who presented with acute anterior uveitis and secondary glaucoma caused by intracameral third stage larvae of Gnathostoma spinigerum. The parasites were successfully removed, with preservation of good visual acuity. The initial infection may occur through consumption of contaminated water and/or raw or undercooked foods, while the eyes may be involved even years later. Accurate diagnosis and surgical removal of the parasites may save life.

Human infestation by the spiruroid nematode, Gnathostoma spinigerum, has been frequently reported in Asia. The usual mode of infection is believed to be ingestion of raw, inadequately cooked or fermented fish or meat. Systemic manifestations include migratory cutaneous swelling and eosinophilic myeloencephalitis. Ocular involvement occurs incidentally to systemic migration of the parasite and may occur years after the initial infection. The increase in tourism to, and refugees from, South-east Asia make it likely that cases of gnathostomiasis will become more frequent in the western hemisphere. We report two cases of intracameral third stage larvae of gnathostoma resulting in acute anterior uveitis and secondary glaucoma. In both cases the parasites were successfully removed and good visual acuity restored. Detection of parasites on ophthalmic examination and surgical removal may prevent further systemic damage and even death.

\section{Case reports}

CASE I

A 61 year-old woman presented with an eight-day history of pain and loss of vision in her left eye. Two weeks previously she had noted a painful cutaneous Correspondence to Somchai Kittiponghansa, MD, Department of Ophthalmology, Faculty of Medicine, Srinagarind Hospital, Khon Kaen University, Khon Kaen 40002, Thailand. swelling on her left hand. The swelling disappeared several days later without treatment.

On examination the right eye was normal. Visual acuity in the left eye was hand movements. Intraocular tension was $40 \mathrm{mmHg}$. The anterior chamber contained a fibrinous exudate. An inactive, curvilinear, living parasite about $5 \mathrm{~mm}$ long was seen at the temporal side of the pupillary border (Fig. 1). Multiple fenestrations caused by parasitic migration were present on the iris surface.

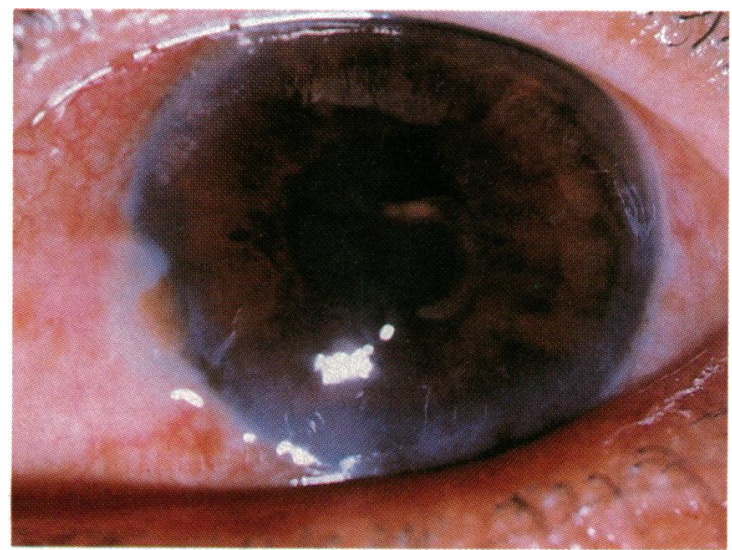

Fig. 1 Case 1. The living parasite about $5 \mathrm{~mm}$ long at the temporal side of the pupillary border. 
The patient was treated with oral glycerin and acetazolamide. The white cell count was $8.8 \times 10^{y} / 1$, with $40 \%$ neutrophils, $51 \%$ lymphocytes, $3 \%$ monocytes, $5 \%$ eosinophils, and $1 \%$ basophils. Urine analysis and chest $x$-ray were normal.

Under general anaesthesia a corneoscleral section of 3 clock hours was made. The worm became active and migrated to the site of the surgical incision, where it was captured by its cephalic portion with lens capsule forceps. Postoperatively the patient was treated with topical dexamethasone, neomycin, and atropine, together with oral acetazolamide and prednisolone $30 \mathrm{mg}$ daily. Her visual acuity improved to $6 / 18$. Fundus examination revealed a normal optic nerve head and no evidence of parasitic tracks in the posterior segment. Over the following year a mature cataract developed in the affected eye.

The living parasite was transported in $0.85 \% \mathrm{NaCI}$ to one of us (SP) for identification. It was fixed in $5 \%$ formalin and mounted in polyvinyl alcohollactophenol. It was identified as the third stage larva of Gnathostoma spinigerum and measured $4.5 \mathrm{~mm}$ long and $0.6 \mathrm{~mm}$ wide (Fig. 2).

\section{CASE 2}

A 32-year-old woman complained of blurred vision and pain in her left eye for one month. Two months previously she had had itching and cutaneous swelling around the left eye. Cellulitis presumptively caused by gnathostoma had been diagnosed and prednisolone $30 \mathrm{mg}$ daily had been given for symptomatic relief.

Examination revealed visual acuity $6 / 60$ in the right eye and $6 / 36$ in the left. The intraocular tension was $17 \mathrm{mmHg}$ in the right eye and $43 \mathrm{mmHg}$ in the left. Old central corneal scars were present bilaterally. The conjunctiva of the left eye was injected and the cornea oedematous. The anterior chamber was clouded by a fibrinous exudate and an immobile, coiled worm approximately $5 \mathrm{~mm}$ long was present in

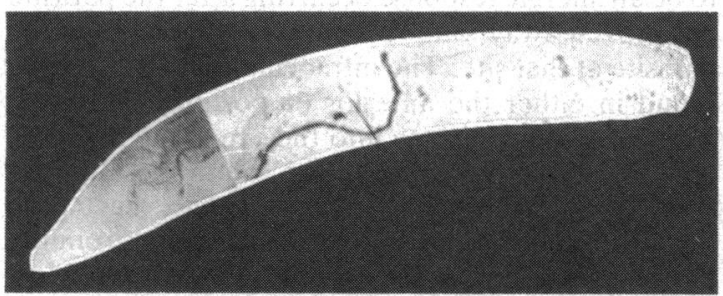

Fig. 2 Case 1. The worm consists of three parts. The stoma is anterior. The second part is the cephalic bulb, measuring $0.20 \mathrm{by} 0.33 \mathrm{~mm}$, and consisting of four rows of single pointed hooklets with rather smaller size in the first row of the anterior end. The third part is the body, consisting of many single pointed cuticular spines in transverse fashion with clearly seen cervical sac along oesophagus.

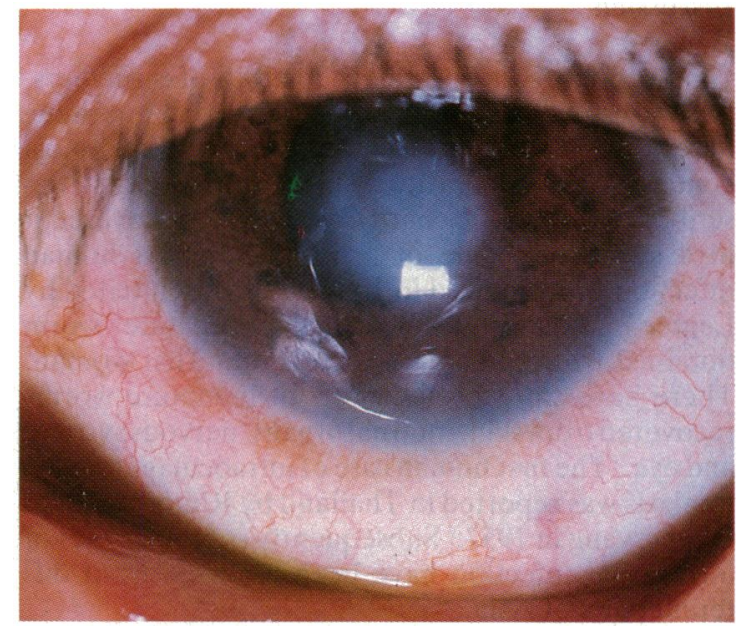

Fig. 3 Case 2. The inactive living parasite in the inferior portion of the anterior chamber.

the inferior portion of the anterior chamber (Fig. 3). Approximately 10 perforating holes caused by parasite migration were noted on the iris.

The patient was treated with oral glycerin and acetazolamide. The white cell count was $11.7 \times 10^{\%} / 1$, with $75 \%$ neutrophils, $14 \%$ lymphocytes, $4 \%$ monocytes, and $7 \%$ eosinophils. Urine analysis and chest $x$-ray were normal.

The parasite was removed from the anterior chamber as in the previous case and sent alive to one of us (SP) for identification. Postoperatively the patient was treated in the same manner as the first patient. Her vision improved to $6 / 24$. The optic nerve head was normal and there was no evidence of a parasitic track. The lens has remained clear for two years.

The worm was processed as in the first case and identified as the third stage larva of $G$. spinigerum, $5.2 \mathrm{~mm}$ long and $0.7 \mathrm{~mm}$ wide. (Fig. 4)

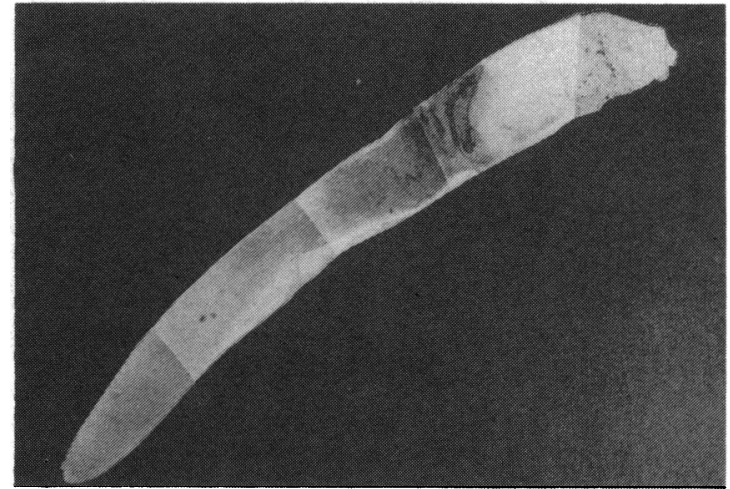

Fig. 4 Case 2. Composite microscopical picture of the third stage larva of $\mathrm{G}$. spinigerum. 


\section{Discussion}

Gnathostoma is an intestinal nematode of carnivores. Gnathostoma spinigerum is the most common tissue parasitic infection in Thailand and the second most common ocular parasite. The worm was first described in 1836 by Richard Owen, who discovered it in a stomach nodule of a tiger'. The first human infection was reported in 1889 by Levinsen (cited by Leiper $^{2}$ ), who studied a single immature worm obtained from the breast abscess of a Thai woman. There have since been reports of immature worms recovered from skin, internal organs, nervous system, and eye. ${ }^{3}$ The first human case of intraocular gnathostomiasis was reported in Thailand by Rhithibaed and Daengsvang in $1957 .{ }^{+}$Subsequently sporadic cases of intraocular infection have been reported from different parts of Asia. ${ }^{5-8}$

The central region of Thailand is one of the most important endemic areas of this parasite in Asia. Much of the food and drinking water in this region is obtained from fresh water rivers and lakes, and intestinal parasitic infestation is common.

The parasite lives encysted in the gastric mucosa of dogs, cats, and wild felines (Fig. 5). The red-orange worm measures about $4.5 \mathrm{~mm}$ long and $0.5 \mathrm{~mm}$ wide. The head bulb contains rows of hooklets and the body contains numerous spines. The ova are passed in faeces and hatch in fresh water to first-stage larvae. These are ingested by the copepod cyclops, the first intermediate host, in which the second-stage larvae develop. The cyclops are in turn eaten by fresh-water fish, frogs, snakes, and eels, the second intermediate hosts, in which the infective third-stage larvae

\section{GNATHOSTOMIASIS Gnathostoma spinigerum}

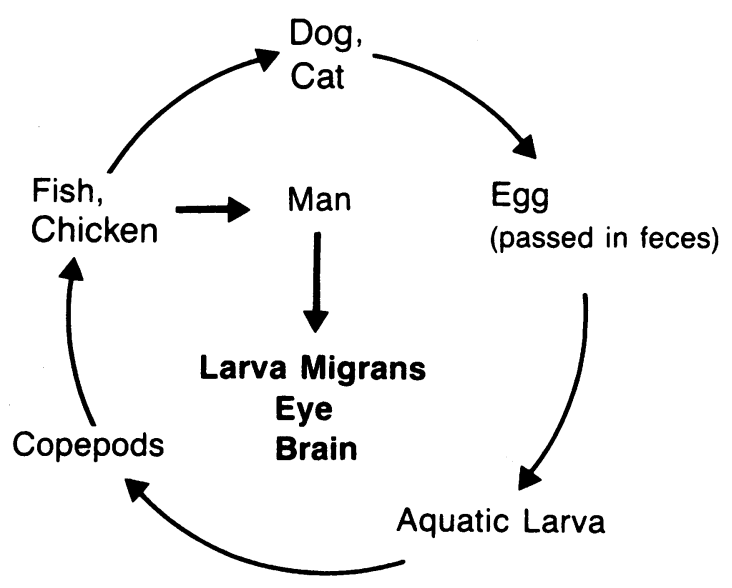

Fig. 5 Life cycle of G. spinigerum. develop. ${ }^{9}$ Vertebrates such as cats and dogs complete the cycle. Man may become a facultative host by eating raw, infected intermediate hosts, such as fresh-water fish, frogs, and snakes. If intermediate hosts are eaten by paratenic hosts, such as ducks and chickens, the third stage larvae cannot undergo further development. They can, however, infect the paratenic hosts and in turn infect man if the paratenic hosts are eaten raw or insufficiently cooked. In Thailand more than 36 species of fresh water fish have been found to be infected with the third-stage larvae."

The infection is more common in women than in men, perhaps owing to transmission via skin penetration during food preparation or from contaminated water containing infected copepods. "The third stage larvae cannot complete their cycle in man, and they migrate for years, causing inflammation in multiple organ systems. ${ }^{12}$

The clinical manifestations of gnathostomiasis include migratory cutaneous swellings, abdominopulmonary hypereosinophilic syndrome, and fatal eosinophilic myeloencephalitis. ${ }^{13-15}$ Damage may occur in any organ due to random migration of the parasite through the body. Recurrent migratory cutaneous swellings and eosinophilia in a patient living in or having travelled to an endemic area make the diagnosis likely. Symptoms often begin in the evening, perhaps due to increasing motility of the worms at this time. Skin testing with the third-stage larval antigen supports the clinical diagnosis when it is positive.

Despite the fact that the north-eastern region of Thailand is a major endemic area of gnathostoma reported cases of intraocular gnathostomiasis are rare. Lid swelling and intraocular parasites are the two major ocular manifestations, and may result in orbital cellulitis, uveitis, hyphaema, vitreous haemorrhage, and central retinal artery occlusion. The lid swelling is accompanied by itching, redness, and pain, which may be mild to severe. It is thought to be an allergic response occurring after the parasite has moved away, and it is useless to search for the parasite at that site. The intraocular parasite may be found in either the anterior or posterior segment. The larvae may migrate into the eye along the optic nerve or directly penetrate the sclera, resulting in traumatic retinal holes, vitreous haemorrhage, and iris perforation. Fibrous bands may extend from the point of entry into and through the vitreous. Most cases are presumptively diagnosed on the basis of the clinical picture, the high percentage of blood eosinophils, and the history of migratory swelling in relation to food habits and residence. Definitive diagnosis rests on the recovery and identification of the parasites. 
Our two patients had the same clinical pictures of acute anterior uveitis with secondary glaucoma but with normal eosinophils. The unusually low eosinophil count in our patients may be due to the fact that the parasites were in the anterior chamber, which is avascular, the cellular response therefore being much less than when the parasites are present in vascular tissues or spaces. The parasites were seen lying inactive in the anterior chamber. However, the numerous iris holes in both cases are suggestive of highly active worms at the initial stage of ocular involvement. In the absence of a visible parasite in the anterior chamber the presence of numerous iris holes can be the basis of diagnosis of parasitic ocular infestation. Anterior uveitis may be present after the parasite has migrated away. The fibrinous exudate and higher intraocular pressure may have led to the cessation of active migratory behaviour and thus to successful removal.

More commonly, removal of the parasite is thwarted by the escape of the parasite from the eye. Freezing the active worm with cryoapplication prior to its removal is usually considered in order to prevent death from systemic migration of the parasite. Failure to remove the parasite may lead to cerebrovascular accident if the parasite migrates into the central nervous system. ${ }^{16}$ To increase the success of removal, ocular manipulation should be kept at a minimum during the procedure. For this reason we favour removal under general anaethesia instead of retrobulbar block. Corneal wetting and anterior chamber irrigation should be avoided as much as possible to minimise activation of the parasite.

There is no specific therapy other than surgical removal. Subcutaneous administration of Ancylol (2,6-diodo, -4 nitrophenol) given subcutaneously has been effective in experimentally infected cats. ${ }^{17}$ Since the parasite can migrate rapidly, immediate surgery is required.

In our cases the parasites were successfully removed with lens capsule forceps without cryoprobe application. Intraocular tissue damage was slight in the first case, as hyphaema from an iris tear. Visual acuities were restored to 6/18 and 6/24 in the first and second patients respectively. However, complicated cataract with subluxation was later observed in the first patient and was attributed to the parasites rather than surgical trauma. The worms removed from the two patients were the third stage larvae.

The source of infective larvae in these cases could be from impure water or pickled fresh water fish.1 The abundance and variety of infected intermediate hosts reinforce the position of parasitic infections as a major health problem of Thailand.

Prevention depends on avoidance or adequate cooking of such food as fresh water fish, snails, and pork. Better sewage disposal and the treatment of drinking water may prevent the spread of parasites in the community.

Gnathostomiasis is rarely diagnosed in Europe and the United States. However, not only may ocular involvement occur several years after the initial infection, but it may be the presenting sign of a previously silent infection. ${ }^{19}$ Increasing world travel, importation of food, and the influx of refugees from South-east Asia demand greater awareness of the parasitology of this region..$^{211}$ Gnathostomiasis should be included in the differential diagnosis of anterior uveitis with or without glaucoma in patients who have travelled to South-east Asia. Multiple iris perforations are diagnostic of a migrating parasite if none can be seen at the time.

$G$ spinigerum may live for years in man, causing larva migrans and perhaps fatal eosinophilic myeloencephalitis. No antiparasitic drugs are available to treat ocular involvement, and therapeutic success depends on early and complete surgical removal. Because the eye is the only site at which direct visualisation and surgical removal are possible, ocular examination is crucial to proper diagnosis and treatment, which may be lifesaving.

This work was supported in part by the Glaucoma Foundation.

\section{References}

1 Owen R. Anatomical description of two species of Entozoa from the stomach of a tiger (Felis tigris $L$ ), one of which forms a new genus of Nematoidea Gnathostoma. Proc Zool Soc Lond 1836; 47: $123-6$.

2 Leiper RT. The structure and relationships of Gnathostoma siamensis (Levinsen). J Parasitol 1909; 2: 72-7.

3 Tansurat P. Human gnathostomiasis. J Med Assoc Thai 1955; 38: 25-32.

4 Rhithibaed C, Daengsvang S. A case of blindness caused by Gnathostoma spinigerum. J Med Assoc Thai 1937; 19: 840-5.

5 Sen K, Ghose N. Ocular gnathostomiasis. Br J Ophthalmol 1945; 29: 618-26.

6 Witenberg G, Jacoby J, Stechelmacher S. A case of ocular gnathostomiasis. Ophthalmologica 1950; 119: 114-22.

7 Gyi K. Intraocular gnathostomiasis. Br J Ophthalmol 1960; 44: 42-5.

8 Bashirullah A. Occurrence of Gnathostoma spinigerum Owen 1836 in Dacca, Bangladesh. J Parasitol 1972; 58: 187-8.

9 Tansurat P. Certain foods containing infective larvae of Gnathostoma spinigerum. J Med Assoc Thai 1945; 27: 79-99.

10 Daengsvang S, Papasarathorn T, Chulalerk U, Tongkoom B. Epidemiological observation on Gnathostoma spinigerum in Thailand. J Trop Med Hyg 1964; 67: 144-7.

11 Daengsvang S. Infectivity of Gnathostoma spinigerum larvae in primates. J Parasitol 1971; 57: 476-8.

12 Bunnag T. Comer DS, Punyagupta S. Eosinophilic myeloencephalitis caused by Gnathostoma spinigerum. Neuropathology of nine cases. J Neurol Sci 1970; 10: 419-34.

13 Sirisamban BS. Report of an eye infection with Tua Chid ( $G$. spinigerum). J Med Assoc Thai 1941; 24: 401-8.

14 Namatra B, Laosuntorn M, Bedavanija A, Lopansri T. Gnathostoma spinigerum in the anterior chamber. J Med Assoc Thai $1962 ; 45: 549-57$. 
15 Punyagupta $\mathrm{S}$. An advanced knowledge about gnathostomiasis. J Med Assoc Thai 1967; 50: 656-94.

16 Boongird P, Phuapradit P, Siridej N. Chirachariyaveij T, Chuahirun S, Vejjajiva A. Neurological manifestations of gnathostomiasis. J Neurol Sci 1977; 31: 279-91.

17 Daengsvang S. Chemotherapy of feline Gnathostoma spinigerum migration stage with multiple subcutaneous doses of ancylol. Southeast Asian J Trop Med Public Health 1980; 11: $359-62$.
18 Daengsvang S. Gnathostomiasis, the life cycle of Gnathostoma spinigerum and methods of transmission. J Med Assoc Thai 1937; 20: 116-46.

19 Tudor RC, Blair E. Gnathostoma spinigerum: an unusual cause of ocular nematodiasis in the western hemisphere. Am J Ophthalmol 1971; 72: 185-90.

20 Teekhasaenee C, Ritch R, Kanchanaranya C. Ocular parasitic infection in Thailand. Rev Infec Dis 1986; 8: 350-6.

Accepted for publication 13 August 1986. 